\title{
Developing a "benefits of parental feeding behaviors" questionnaire for parents of preschoolers in urban Thailand
}

Developing a questionnaire for parents

\author{
Chollada Jongsomjitt, Waraporn Chaiyawat and Branom Rodcumdee \\ Faculty of Nursing, Chulalongkorn University, Bangkok, Thailand
}

Received 17 June 2019 Revised 23 October 2019 26 November 2019 Accepted 25 December 2019

\begin{abstract}
Purpose - Awareness concerning the benefits of an action is a consistent influential predictor of personal behavior and essential for healthcare providers in assessing the benefits of parental feeding behaviors (BePFB) toward preschoolers. Nevertheless, a valid and reliable measure of assessment has yet to be found. The objective of this study was to develop a questionnaire on the BePFB for preschoolers' parents in urban Thailand.

Design/methodology/approach - The instrument development process comprised two phases: scale construction and psychometric property testing. Phase I involved generating an item pool from the literature review and interviews with 17 preschoolers' parents as the measurement format. The items were then reviewed for content validity by five experts, with items selected by evaluating the factor loading and communality score from exploratory factor analysis $(n=310)$. Phase II involved testing the psychometric properties by confirmatory factor analysis (CFA) and reliability using internal consistency $(n=303)$.

Findings - The first draft of the "Benefits of Parental Feeding Behaviors Questionnaire" (BePFBQ) contained 24 items. Five items were deleted by the experts due to redundancy. Six items were then removed after evaluating the item selection process. CFA reported a good fit with the data $\left(\chi^{2} / d f\right.$ ratio $=1.70$, GFI $=0.97$, $\mathrm{CFI}=0.99$, RMSEA $=0.048$ ). The regression weights ranged from 0.50 to 0.74 , at a statistically significant level of 0.05 with an acceptable value for Cronbach's alpha $(a=0.91)$. Finally, the BePFBQ contained 13 items in one dimension, with the 5-point Likert scale.

Originality/value - The BePFBQ is a reliable and valid instrument for measuring the BePFB in preschoolers' parents in urban Thailand.
\end{abstract}

Keywords Parental feeding behaviors, Instrument development, Thailand

Paper type Research paper

\section{Introduction}

Parental feeding behaviors are measured by the specific activities performed by parents toward their children such as providing healthy foods, a pleasant eating environment and enhancing good eating behaviors [1]. As dependent care providers, parents are more likely to perform appropriate feeding behaviors if they know that the children will gain substantial benefit from it. Personal knowledge of the benefits of a specific action is an influential motivation and a consistent predictor of behavior [2,3]. Awareness concerning the benefits of an action relates to the individual's desire or need to exhibit a certain behavior, originating from stimulating forces. Greater awareness of the benefits is likely to motivate more parents to perform appropriate feeding behaviors toward their children [4-7].

(C) Chollada Jongsomjitt, Waraporn Chaiyawat and Branom Rodcumdee. Published in the Journal of Health Research. Published by Emerald Publishing Limited. This article is published under the Creative Commons Attribution (CC BY 4.0) license. Anyone may reproduce, distribute, translate and create derivative works of this article (for both commercial and non-commercial purposes), subject to full attribution to the original publication and authors. The full terms of this license may be seen at http:// creativecommons.org/licences/by/4.0/legalcode

The authors are grateful to the $100^{\text {th }}$ Anniversary Chulalongkorn University Doctoral Scholarship for awarding a research grant to support this study.

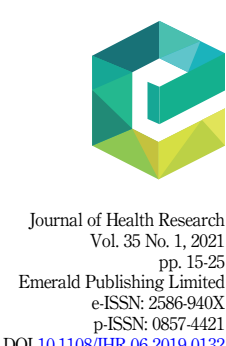

DOI 10.1108/JHR $06-2019-0132$ 
JHR

35,1

\section{6}

Young children with limited ability to take care of themselves require dependent care from parents. Encouraging children to eat appropriately has a significant effect on their eating habits and health in the short and long term. However, existing literature illustrates that Thai preschoolers have poor eating habits, and the rate of obesity is increasing [8], as well as being more prevalent in urban areas such as Bangkok [8, 9]. Parental understanding as to the benefits of feeding behavior is important because it significantly influences its quality.

Benefits of parental feeding behaviors or "BePFB" in this study is a concept derived from "motivation" - one of the power components in Orem's Self-Care Theory in 2001. According to Orem, motivation is goal-oriented toward life, health and well-being [2]. This concept is very important and used by most nurses to help motivate people to perform self-care/dependentcare [10]. Motivation in the nursing context is addressed from the perspective of individuals who engage in activities to bring about conditions within themselves, or the therapeutic selfcare demand of their patients [11]. The benefit has been described as the personal perception or knowledge of the positive consequences of specific behaviors. A theoretical substruction diagram is presented in Figure 1.

In addition, motivation can be described as the tenets of self-determination theory which explains the quality of human functions involving the experience of an internal perceived locus of causality. It is integral to intrinsically motivated behavior and also evident in some extrinsically motivated behavior [12]. Knowledge as to the benefits of an action, and as an internal perception, is a consistent and powerful motivator of a person's behavior [3, 10, 12, 13]. Therefore, the benefits perceived by a person were identified as intrinsic motivation to perform certain behaviors.

In order to facilitate the successful performance of parental feeding behaviors, healthcare providers need to assess and understand the BePFB. To develop the measurement, initial identification as to the definition of the concept is recommended [14]. The BePFB in this study refers to the parents' perception of information concerning the positive consequences for preschoolers of such parental feeding behaviors. Although this concept presents the definition and its construct, the reflective content is not observed. It is thus necessary to study such items.

Many researchers have studied the perceived benefits of various actions based on specific contexts, such as the developmental promotion behavior of toddler mothers [15], promoting the toddlers' health through their mothers' actions [7], such as infection prevention behavior in mothers of 1-5-year-old children with cancer undergoing chemotherapy [16], and the acute respiratory infection prevention behavior of child care workers [17]. However, there was only

Figure 1.

Theoretical substruction diagram

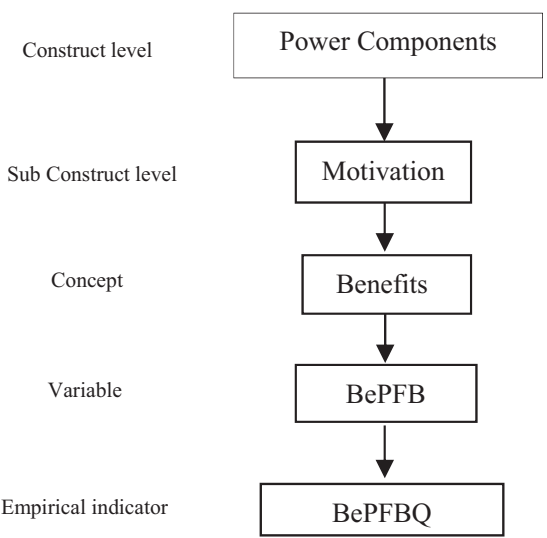


one existing instrument, proposed by Palupi in 2015, who developed a questionnaire on the "Perceived Benefits of Maternal Feeding Behaviors" to measure the perceived benefits of such behavior for toddlers. The original version of this questionnaire was in the Indonesian language, and the English version has not been tested for validity and reliability. Moreover, this instrument was developed for the mothers of toddlers, and some items reflect the emotional feelings of mothers in performing certain actions that were inappropriate for measuring the BePFB. For example, "providing healthy food for my toddler makes me happy," "helping my toddler to enhance good eating behavior lets me have a good relationship with them" and "promoting a pleasant eating environment for my toddler makes me happy" [4]. For this reason, there was no valid and reliable measurement for assessing the BePFB. Therefore, the purpose of this study was to develop an appropriate instrument to measure the BePFB of preschool parents in urban Thailand.

\section{Methodology}

The objective of this study was to develop the Benefits of Parental Feeding Behaviors Questionnaire (BePFBQ). The procedure for developing the BePFBQ was guided by the works of DeVellis [14] and Nunnally and Bernstein [18] and comprised two phases: (1) scale construction and (2) psychometric property testing.

\section{Research setting and participants}

The study was conducted at schools in Bangkok, Thailand, to measure the BePFB. The participants consisted of a father or mother of a preschooler aged 3-6 years studying in Bangkok. The multi-stage random sampling technique was used to recruit participants. The Primary Education Service Area Office in Bangkok was selected for purposive sampling because this organization provides education for students aged 3-6 years. Four schools were recruited using a simple random sampling technique obtained from the website (https://www. random.org). Finally, convenience sampling was used to recruit participants for each phase of this study. A group of 327 participants was recruited for the first phase (scale construction), 17 of whom were interviewed by the researchers, with the remaining 310 recruited to participate in item selection. Moreover, the second phase (psychometric property testing) consisted of 303 participants. A summary of the sample sizes is presented in Table 1.

All data were gathered from September 2018 to March 2019. Participants in the interview group can be characterized as follows: 15 out of the 17 participants were mothers $(88 \%)$ aged from $31-40$ years $(47 \%)$ and $41-50$ years (35\%). Moreover, the majority of participants had only one child $(47 \%)$ or two children $(47 \%)$. Most preschoolers were girls $(70 \%)$. Six preschoolers were aged from $3-4$ years $(35 \%)$ and $4-5$ years $(35 \%)$, while five were aged from5-6 years $(30 \%)$.

Additionally, for the item selection part, $70 \%$ of the 310 participants were mothers. Half were aged from $31-40$ years $(49.2 \%)$ and $20-30$ years (32.8\%). About $55 \%$ of participants had

\begin{tabular}{lll}
\hline Process of instrument development & First and second schools & Third and fourth schools \\
\hline
\end{tabular}

1. Scale construction

1.1 Interviews

1.2 Item selection

17

310

2. Psychometric property testing

$2.1 \mathrm{CFA}$ and reliability

Total
Developing a questionnaire for parents 
JHR

35,1

18

two children, while $43.6 \%$ had only one child, and $55 \%$ of preschoolers in this group were boys. Preschoolers were aged $3-4$ years $(19.9 \%), 4-5$ years $(36.9 \%)$ and $5-6$ years $(43.2 \%)$.

Lastly, 303 participants were recruited for the second phase, the majority of whom were mothers (74\%) aged from 31-40 (56.5\%) and 20-30 (25.5\%). Half had two children (49.8\%), while $35 \%$ had only one child. The preschoolers were aged from 3-4 years (43\%), 4-5 years $(37 \%)$ and $5-6$ years $(20 \%), 52 \%$ of whom were boys.

\section{Procedures and ethical issue}

The procedure for instrument development was run following approval from the Ethical Review Committee for Research Involving Human Research Subjects, Health Science Group, Chulalongkorn University, Thailand (COA No 0.230/2561).

The director and subsequently the kindergarten teachers at each school were contacted for permission to collect data. Participants were selected using the convenience sampling method and contacted following the teachers' suggestions. Informed consent forms were signed by the parents to confirm their acceptance to participate in the study. The instrument development procedure for the BePFBQ consists of two phases as follows.

\section{Phase I: scale construction}

The researchers began by generating an item pool from a broad review of the existing literature. Previous research on the BePFB in urban Thailand was found to be limited. Most of the existing literature consisted of studies conducted in other countries. Therefore, a qualitative study involving interviews with the preschoolers' parents was required to generate items related to the BePFB in urban Thailand. A broad review of the literature on the $\mathrm{BePFB}$ was undertaken. Data reflecting the parents' perception of information concerning the positive consequences for preschoolers of such parental feeding behaviors were listed and recorded. All data were gathered from existing evidence, such as textbooks, academic articles, academic research and existing instruments. The content of each item had to primarily reflect the construct of interest [14]. The examples of the data obtained are illustrated in the Result section.

The interviews were partially aimed at exploring the parents' perception of information concerning the positive consequences for preschoolers of such parental feeding behaviors. A qualitative study using in-depth, face-to-face interviews was adopted for this phase. Convenience sampling was applied to recruit the first three parents of preschoolers from two schools. Snowball sampling was then used to recruit further participants by the original group inviting parents among their acquaintances. Each participant was interviewed once, over a period of 30-45 minutes, until no new information was obtained. The interview locations, such as a room at a school or coffee shop, were convenient, suitable, safe, quiet and comfortable for both the interviewees and interviewer.

The participants were asked general open-ended questions with no pre-identified concepts [19]: "Could you tell me about taking care of your child regarding food? Please give me an example from a typical day." The participants were then asked probing questions to provide reasons for the activities given to their preschoolers: "Could you please tell me the benefits of this action?," "Do you have any more information to tell me?" and "Is there anything else I should know?" All data concerning the BePFB gathered from interviews were transcribed from the audio recording. Any statements in the participants' narratives relating directly to the phenomenon under investigation were considered and numerically entered into a list. The example data were illustrated in the results section. The data were obtained from 17 participants and is an acceptable sample size in qualitative research $(n>15)$ [20].

Based on the findings of item generation from the in-depth interviews and literature review, an item pool was obtained to compare similarities and differences. Content validity 
was then used to determine the quality and relevance of the items for inclusion in the instrument. According to Downs [21], a minimum of five experts would provide a sufficient level of control for change agreement. The first draft of the BePFBQ was investigated for content validity by five experts experienced in pediatric nursing, instrument development and health promotion in young children. The experts were asked to evaluate the relevance of each item and the objective of the measure using a four-point rating scale from 1 (not relevant) to 4 (very relevant). The content validity index (CVI) of the instrument was calculated based on the number of experts giving a rating greater than 3, divided by the number of experts [22]. Moreover, the experts were asked to explain their reasons if they disagreed with any of the items.

The item selection was then evaluated to identify appropriate items using corrected item-total correlation, inter-item correlation and factor loading with the commonality score run by exploratory factor analysis (EFA). This study used EFA to examine and determine items with a lower score criteria. The principal component method was also applied. Factors were extracted using the maximum likelihood method and rotated by varimax rotation [14]. The acceptable sample size estimate for factor analysis should be at least 300 [18]. Thus, a group of 310 participants was recruited using convenience sampling for item selection. Prior to recruitment, outlier testing presented that no samples were deleted because the absolute $Z$-score values were lower than 3.0 [23]. The acceptable value of the corrected item-total correlation was greater than 0.30 [24], and inter-item correlation should be between 0.20 and 0.80 [18]. Items had a value of inter-item correlation with others higher than 0.80 were considered to select one. Whereas items that had a value of inter-item correlation with others lower than 0.20 or a corrected item-total correlation value greater than 0.30 were considered for deletion.

EFA was applied in this study for item selection. The assumptions of the EFA were tested: normality showed that the skewness ranged from -0.38 to 1.30 and kurtosis from -0.73 to 1.82 , which were acceptable $(-2 \leq$ skewness $\leq 2,-7 \leq$ kurtoses $\leq 7)$ [25]. Principal component analysis (PCA) was used to identify complex interrelationships among individual and group items forming part of the unified concepts [14] with communalities used to select items. Moreover, items loaded on the wrong factor were excluded. With a sample size of between 250 and 349, the criterion for factor loadings had to be greater than 0.35 [26]. Moreover, the commonality score for each item had to be greater than 0.20 [27]. Collinearity was tested using the Kaiser-Meyer-Olkin (KMO) test, with acceptable values greater than 0.5 , and a statistically significant Bartlett's test of sphericity $($ sig $<0.05)$ indicated that sufficient correlation existed among the variables in order to proceed.

\section{Phase II: psychometric property testing}

The second draft of the BePFBQ was examined for construct validity using confirmatory factor analysis (CFA) in SPSS AMOS (version 22) and internal consistency for reliability. The generally agreed lower limit for Cronbach's alpha was 0.70 [26], while the acceptable estimated sample size for factor analysis was at least 300 [18]. Thus, 305 participants not previously involved in this study were recruited. Two participants were then excluded because outlier testing reported that two samples should be removed $(Z$-score $=-3.44$, -3.44). Thus, 303 participants were retained. Item correlation and corrected item-total correlation were evaluated before analyzing the CFA. The values of inter-item correlation ranged from 0.20 to 0.80 , and the value of corrected item-total correlation ranged from 0.46 to 0.70 , which was acceptable. CFA assumption testing revealed that the skewness and kurtosis ranged from -1.599 to -0.111 and -1.791 to 4.983 , respectively. No items were deleted. The KMO was 0.90 and Bartlett's test of sphericity showed a significance of 0.000 , meaning that CFA could be applied to the obtained dataset.
Developing a questionnaire for parents

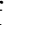


JHR

35,1

20

\section{Results}

Scale construction

Data gathered from the literature review and interviews are presented in Table 2.

An item pool was obtained to compare similarities and differences. The first draft of the BePFBQ contained 24 items in one dimension, using a 5-point Likert scale from 1 (strongly disagree) to 5 (strongly agree). Following review by the experts, the content validity index/ average (S-CVI/Ave) of the scale was found to be 0.84 , lower than the acceptable value (S-CVI/ Ave $\geq 0.90$, I-CVI $\geq 0.80$ ) [22]. Five items with a score lower than 0.80 were then deleted because of redundancy. Therefore, the S-CVI of 19 items subsequently increased to 0.92 . The item selection was then evaluated, and six items removed following the presented criteria. Finally, 13 items for the BePFBQ were retained.

Next, collinearity was tested using the KMO with Bartlett's test of sphericity for statistical significance. The results of the KMO measure for 13 items was 0.93, and Bartlett's test of sphericity was significant at $0.000\left(\chi^{2}=3335.53\right)$, which was adequate for the relationship between items, indicating appropriateness for factor analysis [14]. The results of the EFA with one component presented Eigenvalues above 1.0, accounting for $62.68 \%$ of the variance. The value of Cronbach's alpha coefficient was 0.95 . The results showed that all 13 items could be retained in the BePFBQ (Table 3).

\section{Psychometric property testing}

Construct validity. Confirmatory factor analysis (CFA) was used to examine whether a particular factor model provided a good fit with the data. The results reported regression weights ranging from 0.50 to 0.74 , at a statistically significant level of 0.05 . Moreover, the threshold criteria included the number of observations and observed variables in deciding the cut-off values for model fit. The model was re-specified based on suggestions from the modification indices. The fit indices met the model requirements of multiple criteria following the recommendation by Pedhazur and Schmelkin [24] and Hair et al. [26]. The fit statistics for the BePFBQ model were as follows: chi-square $(\chi 2)=71.29, p=0.000, d f=42, \chi^{2} / d f$ ratio $=1.70$, goodness-of-fit index $(\mathrm{GFI})=0.97$, comparative fit index $(\mathrm{CFI})=0.99$, root-meansquare error of approximation (RMSEA) $=0.048$ (Table 4). The standardized modified measurement of the BePFBQ model was illustrated in Figure 2.

Table 2.

Example data for item generation (from interviews and literature review)
Data from literature

(1) Appropriate preparation of healthy food for children can improve their health and prevent illness [28-30]

(2) Providing healthy food for children helps them to be healthy $[31,32]$

(3) Training the child to use a serving spoon when eating with others can exhibit healthy eating behavior [28, 31, 33, 34]

(4) Providing age-appropriate and healthy food for my child makes my child have good health $[4,29$, 30, 33]

(5) Giving healthy food to the child can promote good health and avoid malnutrition $[5,30]$

(6) Providing healthy food can protect my child from certain diseases $[4,35,36]$

Note(s): $\mathrm{PN}=$ Participant number
Data from interview

(1) Teaching my child to know about the benefits of eating fruits and vegetables encourages them to try more (PN. 3, 4, 8, 9, 13, 14, 15 and 16)

(2) Eating on time can prevent my child against gastritis (PN. 4, 5, 10, 11, 14 and 15)

(3) Preparing plenty of food for my child can maintain her/his nutrition (PN. 1-17)

(4) Making the food at an appropriate size helps children to eat easily and prevents it from getting stuck in their throats (PN. 3, 4, 5, 8-12, 14 and 15)

(5) Not allowing the child to eat sweets can prevent obesity (PN. 2, 5, 10, 11, 15 and 16)

(6) Providing fruits and vegetables for children can prevent constipation (PN. 5, 13 and 16) 
Reliability. The reliability of the BePFBQ was determined by internal consistency analysis using the Cronbach's alpha coefficient. The results showed that the BePFBQ had an acceptable Cronbach's alpha of 0.91 .

To summarize, the BePFBQ contains 13 items in a single dimension. All items were measured using a 5-point Likert scale: 1 (strongly disagree), 2 (disagree), 3 (unsure), 4 (agree), 5 (strongly agree). The rating scores were summated with the total scores, ranging from 1365. A higher score indicates greater perception by parents of the positive consequences for preschoolers as a result of their appropriate feeding behaviors. Details of the BePFBQ are presented in Table 5.

\section{Discussion}

The objective of this study was to develop a BePFBQ for measuring the knowledge level of preschool children's parents in urban Thailand about the positive consequences of developing positive parental feeding behaviors. The BePFBQ consists of 13 items in a single dimension, using a 5-point Likert scale, explained as intrinsic motivation and identified by Deci and Ryan's self-determination theory [12]. Concerning the validity and reliability scale, the BePFBQ was conducted using content and construct validity and internal consistency. A Cronbach's alpha coefficient of 0.91 was obtained for this scale.

The construct validity of the BePFBQ was examined by applying CFA. The results showed that the fit indices were in acceptable ranges $[24,26]$. Even though the results reported an unacceptable value for the $\chi^{2}$ test $(\phi=0.00)$, it was less meaningful in instances of large sample sizes or the number of observed variables [37]. The BePFBQ involved a large sample size. Thus, the normed fit chi-square $\left(\chi^{2} / d f\right)$ was considered instead with a significance of $\chi^{2}$. The value of $\chi^{2} / d f$ was lower at 2 , GFI $>0.90$, CFI $>0.90$ and the RMSEA $<0.05$, which was within the acceptable recommended threshold. All regression weights were significant $(p<0.05)$, demonstrating the construct validity of the BePFBQ and indicating that the model in this study was accepted as being a good fit.

The structural domain in this study contains only one dimension, namely, intrinsic motivation. Intrinsic motivation in this study was used for the term "benefits of parental feeding behavior," a concept substructed from "motivation" which is one of the power components in Orem's self-care theory. The literature indicates that awareness concerning the benefits of a person's behavior results in an innate propensity to pursue their intrinsic

\begin{tabular}{|c|c|c|c|c|c|c|}
\hline Total item & Communalities & Factor loading & Eigenvalue & Cumulative $\%$ & Cronbach's alpha & Results of the BeFPBQ \\
\hline 13 & $0.50-0.81$ & $0.36-0.90$ & 8.148 & 62.68 & 0.95 & $\begin{array}{l}\text { exploratory factor } \\
\text { analysis }(n=310)\end{array}$ \\
\hline
\end{tabular}

\begin{tabular}{lcc}
\hline & & Values \\
Goodness of fit statistics & Threshold (reference) & Study results \\
\hline$\chi^{2}$ & $(p>0.05)$ & $71.29(p=0.003)$ \\
df & - & 42 \\
$\chi^{2} / \mathrm{df}$ & $<2$ & 1.70 \\
CFI & $>0.90$ & 0.99 \\
GFI & $>0.90$ & 0.97 \\
RMSEA & $<0.05$ & 0.048
\end{tabular}

Note(s): The level of significance was set at the 0.01 (two-tailed)
Developing a questionnaire for parents 


\section{JHR 35,1}

\section{2}

Figure 2.

Standardized modified measurement model for the BePFBQ

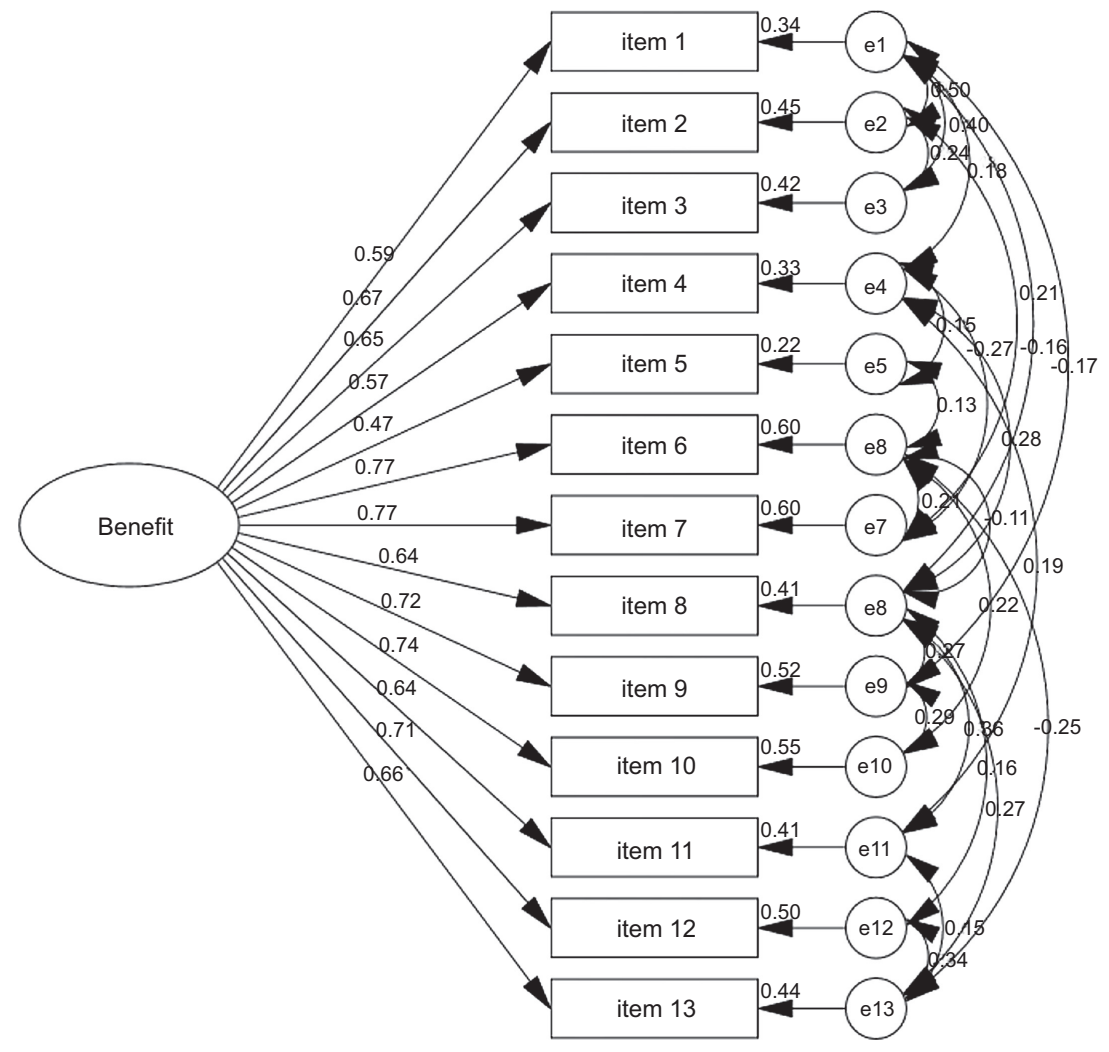

Chi-square $=71.290, \mathrm{df}=42, P=0.003, \mathrm{CMIN} / \mathrm{DF}=1.687$, $\mathrm{CFI}=0.987, \mathrm{RMSEA}=0.048, \mathrm{SRMR}=0.011$

Item Data

1 Encouraging the child to eat vegetables/fruits every day can prevent constipation

2 Looking after the child while they are eating can help them receive sufficient nutrition

$3 \quad$ Providing nutritious food makes the child healthy

4 Ensuring the child eats an adequate amount of food can prevent childhood obesity

5 Allowing the child to eat strong flavors, such as food that is too sour or spicy, causes a negative effect on the stomach, intestines and digestive system

$6 \quad$ Ensuring the child eats freshly cooked food can prevent germs from forming and causing diarrhea

$7 \quad$ Ensuring the child eats on time can prevent gastritis

8 Teaching the child to know the benefits of eating vegetables/fruits makes the child more interested in eating them

9 Training the child to use a serving spoon when eating with others can prevent communicable diseases

10 Teaching the child to wash their hands before and after eating can prevent germs

Table 5. $\begin{array}{lll}\text { The Benefits of } & 11 & \text { Teaching the child to eat various kinds of foods can deter them from choosing the same food repeatedly } \\ \text { Parental Feeding } & 12 & \text { Providing foods in an appropriate size for eating, and ensuring it is not too sticky or too hard can help }\end{array}$ Behavior Questionnaire (13 items)

13 Providing brightly colored vegetables which are not pungent, soft or crispy can motivate the child to eat more vegetables 
needs by expressing such behavior. Every parent in this study acknowledged the beneficial outcomes of positive parental feeding behaviors (children remain healthy and have good eating habits). In this study, the BePFB contained only one category. In previous literature, no instruments for benefiting parental feeding behavior had been identified. However, other populations have assessed the benefits of specific behavior. For instance, the "Benefits of Maternal Feeding Behaviors Questionnaire" [4] contains one dimension and measures maternal perceptions when performing appropriate feeding behavior toward toddlers. Even though the instrument in this study has one dimension, some items reflect the emotional feelings of mothers in performing certain actions, such as "providing age-appropriate and healthy foods for my toddler makes me happy," in contrast to the BePFBQ in this study.

Moreover, a study on the perceived benefits of eating a healthy food scale [38] was conducted to measure the effects on African-American people, using only one dimension as in the BePFBQ for the present study. However, the perceived benefits of eating a healthy food scale were developed using the health promotion model by Pender [1], and some items reflected the emotional feelings of mothers in performing certain actions. For example, "eating healthy foods would help me feel better," in contrast to the BePFBQ in the present study.

Similarly, the "Perceived benefits of health promotion for toddlers questionnaire" [7] was conducted to measure the perception of benefit by mothers from toddlers eating healthily, using only one dimension as in the BePFBQ developed for this study. The items in the "Perceived benefits of health promotion for toddlers questionnaire" reflected the awareness of mothers concerning the beneficial outcomes for their toddlers; for example, "taking care of my child when brushing their teeth helps to keep their teeth clean." However, the "Perceived benefits of health promotion for toddlers questionnaire" was conducted using Pender's Health Promotion Model, in contrast to the BePFBQ in this study.

The term "motivation" was found in various theories such as the self-care theory [2], the health promotion model [3] and the self-determination theory [12]. This study uses the selfdetermination theory since it suggests that humans have an innate propensity to pursue their intrinsic needs by expressing their behavior. Importantly, the self-determination theory also highlights the role of society, which can facilitate intrinsic motivation by functioning optimally when a child's need for autonomy is supported by their parents [12].

This current study shows that the BePFBQ is a reliable and valid measure for assessing the benefits of parental feeding behavior for preschoolers in urban Thailand. However, this instrument was first developed for use in the Thai context, especially urban areas, and does not include rural areas and different populations. Further study should include a normreferenced test. In addition, the samples in this study consisted of parents of preschoolers attending schools associated with the Primary Education Service Area Office in Bangkok. Future studies should be conducted in diverse settings, such as schools under the Office of the Higher Education Commission, Office of Private Education Commission, Local Administrative Organization and Department of Education.

The BePFBQ will be useful in practice and for future research. It will provide pediatric nurses and other healthcare professionals with an accurate measure for assessing awareness concerning the benefits of parental feeding behavior for preschoolers in urban Thailand. Parents with greater awareness of the benefits tend to perform more appropriate parental feeding behavior toward their preschoolers. Ultimately, such behavior can promote the health of preschoolers and encourage good eating habits.

In conclusion, the BePFBQ was developed for use by preschooler parents in urban Thailand. This instrument could be used as a measurement for awareness concerning the benefits of parents performing appropriate feeding behaviors toward preschoolers. The BePFBQ could be used as an intervention to promote parental feeding behavior for

Developing a questionnaire for parents 
JHR 35,1 knowledge improvement by providing information or alternative methods of behavior for preschooler parents.

\section{References}

1. Lusmilasari L. The development of the parental feeding behaviors questionnaire (PFBQ) for Indonesian parents with toddlers. Bangkok: Chulalongkorn University; 2014.

2. Orem DE. Nursing: concepts of practices. 6th ed. St. Louis: Mosby; 2001.

3. Pender NJ, Murdaugh CL, Parsons MA. Health promotion in nursing practice. 6th ed. Boston, MA: Pearson; 2006.

4. Palupi E. Selected factors predicting maternal feeding behaviors for toddlers, Java Island, Indonesia. Bangkok, Thailand: Chulalongkorn University; 2014.

5. Sitthideth D. Perception of mothers on child feeding practice and prevention of malnutrition among children of 6 - 59 months of age in Sepol district, Savannakheth province, laos. National Institute of Public Health. 2013; 15: 14-5.

6. Hart KH, Herriot A, Bishop JA, Truby H. Promoting healthy diet and exercise patterns amongst primary school children: a qualitative investigation of parental perspectives. J Hum Nutr Diet. 2003 Apr; 16(2): 89-96. doi: 10.1046/j.1365-277x.2003.00429.x.

7. Sakdapetchsiri J. Factors influencing maternal behaviors in promoting toddlers' health at Rayong province. Nakhon Prathom province: Mahidol University; 2002. [in Thai].

8. National Statistical Office [NSO]. The situation of Thai children and women survey 2015-2016. Bangkok: National Statistical Office, Ministry of Digital Economy and Society; 2017.

9. Pediatric Society of Thailand. Guideline in child health supervision. Bangkok: Sapasan; 2014.

10. Hanuchareonkul S. Self-care: sciences and art in nursing. Bangkok: V.J. printing; 1997. [in Thai].

11. Renpenning KM, Taylor SG. Self-care theory in nursing. New York: Springer; 2003.

12. Deci EL, Ryan RM. Intrinsic motivation and self-determination in human behavior. New York: Plenum; 1985.

13. Thanavaro JL, Moore SM, Anthony M, Narsavage G, Delicath T. Predictors of health promotion behavior in women without prior history of coronary heart disease. Appl Nurs Res. 2006 Aug; 19(3): 149-55. doi: 10.1016/j.apnr.2005.07.006.

14. DeVellis RF. Scale development: theory and applications. 3rd ed. Thousand Oaks, Calif: SAGE; 2012.

15. Manowong W. Perceived benefit, self-efficacy and developmental promotion behaviors among mothers of toddlers. Chiang Mai, Thailand: Chiang Mai University; 2007. [in Thai].

16. Jansong $\mathrm{W}$. The effect of the nursing intervention based on pender's health promotion model on infection prevention behaviors in mothers of 1-5-year-old children with cancer undergoing chemotherapy. Bangkok: Chulalongkorn University; 2015. [in Thai].

17. Jaide C, Santati S, Kongsaktrakul C. Factors related to child care workers' behavior in prevention of acute respiratory infection in child care centers. Rama Nurs J. 2012; 18(3): 389-403. [in Thai].

18. Nunnally JC, Bernstein IH. Psychometric theory. 3rd ed. New York: McGraw-Hill; 1994.

19. Corbin J, Strauss A. Basic of qualitative research: techniques and procedures for developing grounded theory. 3rd ed. Thousand Oaks, Calif: SAGE; 2008.

20. Guest G, Bunce A, Johnson L. How many interviews are enough?: an experiment with data saturation and variability. Field Methods. 2016; 18(1): 59-82. doi: 10.1177/1525822x05279903.

21. Downs SF. Readings in research methodology. 2nd ed. Philadelphia: Lippincott; 1999.

22. Polit DF, Beck CT. Essentials of nursing research: appraising evidence for nursing practice. 7th ed. Philadelphia: Lippincott Williams and Wilkins; 2008. 
23. Barnett V, Lewis T. Outliers in statistical data. 3rd ed. Chichester: John Wiley and Sons; 1994.

24. Pedhazur EJ, Schmelkin LP. Measurement, design, and analysis: an integrated approach. Hillsdale, NJ: Lawrence Erlbaum Associates; 1991.

25. Kim HY. Statistical notes for clinical researchers: assessing normal distribution (2) using skewness and kurtosis. Restor Dent Endod. 2013 Feb; 38(1): 52-4. doi: 10.5395/rde.2013.38.1.52.

26. Hair JF, Black WC, Babin BJ, Anderson RE. Multivariate data analysis. 7th ed. Harlow, Essex: Pearson; 2014.

27. Child D. The essentials of factor analysis. 3rd ed. New York: Continuum; 2006.

28. Pocock M, Trivedi D, Wills W, Bunn F, Magnusson J. Parental perceptions regarding healthy behaviours for preventing overweight and obesity in young children: a systematic review of qualitative studies. Obes Rev. 2010 May; 11(5): 338-53. doi: 10.1111/j.1467-789X.2009.00648.x.

29. Carnell S, Cooke L, Cheng R, Robbins A, Wardle J. Parental feeding behaviours and motivations. A qualitative study in mothers of UK pre-schoolers. Appetite. 2011 Dec; 57(3): 665-73. doi: 10.1016/j. appet.2011.08.009.

30. Chotibang J, Fongkaew W, Mo-suwan L, Meininger JC, Klunklin P. Development of a family and school collaborative (FASC) program to promote healthy eating and physical activity among school-age children. Thai J Nurs Res. 2009; 13(2): 133-47. [in Thai].

31. Maneeton C, Wattana T, Banjongpru Y, Leela S, Banjongpru H. Eating behaviors of pregnancy, women giving breastfeeding, infants, and preschoolers in Rural, Eastern Thailand. A workshop of the effect of causes and factors on Thai eating behaviors in 1986. Bangkok: Institute of Nutrition, Mahidol University; 1986. [in Thai].

32. Nicklaus S. Development of food variety in children. Appetite. 2009 Feb; 52(1): 253-5. doi: 10.1016/j. appet.2008.09.018.

33. Nepper MJ, Chai W. Parents' barriers and strategies to promote healthy eating among school-age children. Appetite. 2016 Aug; 103: 157-64. doi: 10.1016/j.appet.2016.04.012.

34. Satter E. The feeding relationship. in: Kessler DB, Dawson P, editors. Failure to thrive and pediatric undernutrition: a transdisciplinary approach. Baltimore: Brookes; 1999: 121-49.

35. Berlin KS, Davies WH, Lobato DJ, Silverman AH. A biopsychosocial model of normative and problematic pediatric feeding. Child Health Care. 2009; 38(4): 263-82. doi: 10.1080/ 02739610903235984.

36. Vereecken C, Maes L. Young children's dietary habits and associations with the mothers' nutritional knowledge and attitudes. Appetite. 2010 Feb; 54(1): 44-51. doi: 10.1016/j.appet.2009. 09.005 .

37. Burton SJ, Sudweeks RR, Merrill PF, Wood B. How to prepare better multiple-choice test items: guidelines for University Faculty. Provo, Utah: Brigham Young University Testing Services and the Department of Instructional Science; 1991.

38. Pawlak R, Colby S. Benefits, barriers, self-efficacy and knowledge regarding healthy foods; perception of African Americans living in eastern North Carolina. Nutr Res Pract. 2009 Spring; 3(1): 56-63. doi: 10.4162/nrp.2009.3.1.56.

\section{Corresponding author}

Chollada Jongsomjitt can be contacted at: mod_tanoy23@hotmail.com

\section{Developing a questionnaire for parents}

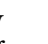

